\title{
Efectos metálicos "nobles" obtenidos mediante el desarrollo de tintas acuosas inkjet a través del empleo de materias primas cerámicas tradicionales
}

\author{
COLORONDA, S.L. \\ Avda. Real Extremadura, 11-13, 12200 Onda (Castellón) España
}

Este trabajo ha participado en la 38 edición de los premios Alfa de Oro, otorgados por la Sociedad Española de Cerámica y Vidrio, durante la Feria Internacional de Cerámica de Valencia CEVISAMA 2014

\begin{abstract}
Coloronda, S.L. ha abordado la viabilidad técnica del desarrollo de tintas de inyección digital de naturaleza acuosa (con un tamaño de partícula inferior a $5 \mu \mathrm{m}$ ), con el objetivo de obtener efectos metálicos nobles a partir de materias primas tradicionalmente utilizadas por el sector cerámico. Para ello, se ha estudiado la influencia de las principales variables y condiciones de trabajo (tratamiento térmico, temperatura, distribución granulométrica, tamaño partícula, composición química, etc.) sobre los mecanismos de nucleación y cristalización de las materias primas utilizadas, siendo éstos los responsables de los fenómenos ópticos producidos sobre baldosas cerámicas obtenidas por monococción.
\end{abstract}

Palabras clave: inyección tinta, tintas acuosas, efectos metálicos nobles, dorado, plateado, efecto cerámico monococción digital.

"Noble" metallic effects obtained by means of the development of water-based inkjet inks using traditional ceramic raw materials

Coloronda, S.L. has tackled the technical viability of developing water-based inkjet inks (containing up to 5- $\mu \mathrm{m}$ particle sizes) so as to obtain noble metallic effects by means of different raw materials traditionally used in the ceramic tile manufacturing process. In this context, several parameters and working conditions (i.e. heat treatment, temperature, particle size, chemical composition, etc.) have been studied in order to examine their influence on the nucleation and crystallization mechanisms which are responsible for the physical-optical phenomena obtained on single-firing ceramic tiles.

Keywords: inkjet, water-based inks, noble metallic effects, golden, silver, single-firing digital effect.

\section{INTRODUCCIÓN}

Como respuesta a las necesidades y requerimientos, cada vez más patentes, suscitados por las entidades integradas en el tejido empresarial para el desarrollo de productos cerámicos de elevado valor añadido, en el presente trabajo se recoge la investigación llevada a cabo por la entidad COLORONDA, S.L. sobre el desarrollo de efectos basados en metales preciosos $(\mathrm{Au}, \mathrm{Ag}, \ldots)$ obtenidos a partir de tintas acuosas inkjet (tamaño de partícula inferior a $5 \mu \mathrm{m}$ ) mediante la utilización de materias primas cerámicas (fritas, plastificantes, desgrasantes, etc.).

En concreto, el desarrollo de nuevos efectos decorativos basados en sistemas formados por partículas vítreas (fritas), así como de sistemas complejos constituídos por componentes de naturaleza tanto vítrea como cristalina (en adelante, multicomponentes), quedaba restringido para su empleo en otros sistemas de decoración cerámicos distintos del propuesto en la presente investigación (serigrafía, flexografía, huecograbado, etc.). Sin embargo, y de acuerdo con los grandes avances tecnológicos realizados en el campo de la impresión inkjet, queda más que justificada la investigación que aquí se propone en vistas a alcanzar una consolidación importante no sólo en la obtención de productos cerámicos con elevadas propiedades estéticas, sino también para el desarrollo de productos capaces de cubrir elevados requerimientos técnicos (dureza superficial, resistencia a la abrasión, resistencia química, etc.).

Así pues, es ampliamente reconocida la dificultad que entraña la obtención de efectos cerámicos a partir de materiales que presentan tamaños de partículas próximos a $1 \mu \mathrm{m}$. De acuerdo con las investigaciones llevadas a cabo en este campo de estudio, conocer y predecir el efecto que produce aquella variable sobre los mecanismos y procesos de nucleación y cristalización de diversas materias primas durante las etapas de sinterización, se convierte en una tarea esencial en favor del valor añadido de los sustratos cerámicos (Prado et alt., 2003; Randall et alt., 1998).

De hecho, el tamaño y la distribución granulométrica de las materias primas empleadas en la elaboración de cualquier esmalte cerámico, ejerce una gran influencia sobre el proceso de sinterización. De este modo, a medida que 
disminuye el tamaño de partícula y se estrecha su distribución granulométrica, se produce una reducción en la temperatura a la que tiene lugar el inicio de la etapa de sinterización, como consecuencia del incremento de la presión capilar (fuerza impulsora del proceso) originada en el interior de los poros y en los puntos de contacto entre partículas. (Escribano et alt., 2001; Siligardi et alt., 2000; Randall et alt., 1998; Gribb et alt., 1997; Tozzi, 1992; Cable, 1991)

De acuerdo con todo lo anteriormente expuesto y teniendo en cuenta la naturaleza superficial de la aplicación inkjet, será necesario tener en consideración la elevada superficie específica que presentan partículas de pequeño tamaño, así como su refractariedad, con la finalidad de reducir y, en el mejor de los casos, evitar los procesos de corrosión química a las que éstas se encuentran sometidas como consecuencia de la formación de las fases líquidas durante la etapa de tratamiento témico de soportes y esmaltes cerámicos; hecho que dificulta la generación de fases cristalinas durante la etapa de sinterización (Rawson, 1991; Fernández, 1991).

\section{FINALIDAD DE LA INVESTIGACIÓN}

Mediante la consecución de la presente investigación ha sido posible:

- Conocer y comprender en profundidad los procesos de nucleación y cristalización de materias primas que tienen lugar durante el proceso de tratamiento térmico de sustratos cerámicos fabricados por monococción, destinados al revestimiento y pavimento de superficies.

- Estudiar la influencia que ejerce el tamaño y la distribución granulométrica de las partículas sobre las etapas que intervienen durante el proceso de sinterización de soportes y esmaltes cerámicos.
- Desarrollar tintas acuosas inkjet (tamaño inferior a 5 $\mu \mathrm{m})$ formadas por múltiples componentes de naturaleza diversa (cristalina y vítrea) que permitan su implantación industrial con plenas garantías.

\section{DESARROLLO EXPERIMENTAL}

Desde Coloronda se ha trabajado en el desarrollo de tres tintas acuosas inkjet capaces de proporcionar efectos metálicos "nobles" sobre soportes cerámicos fabricados por monococción.

- Tinta acuosa inkjet que proporciona Efecto CLASSIC GOLD.

- Tinta acuosa inkjet que proporciona Efecto CLASSIC SILVER.

- Tinta acuosa inkjet que proporciona Efecto CLASSIC OLDEN.

\section{a. Efecto CLASSIC GOLD}

Consiste en el desarrollo de un efecto óptico sobre la superficie de los recubrimientos vidriados de las baldosas cerámicas de monococción, capaz de confirir de un brillo metalico que cambia de tonalidad al modificarse el ángulo de incidencia de la luz, para proporcionar un aspecto dorado a las decoraciones.

Dicho efecto es posible, gracias al empleo de un material fundente que acelera el proceso de sinterización y maduración, desplanzadoel intervalo de temperaturas de cocción hacia valores más bajos. De esta forma es posible la disolución e incorporación en el masa fundida de distintos materiales refractarios utilizados en la formulación de la tinta. La desvitrificación producida durante la etapa de tratamiento térmico permite la generación de cistales orientados sobre la superficie a decorar, siendo éstos los responsables del efecto desarrollado.

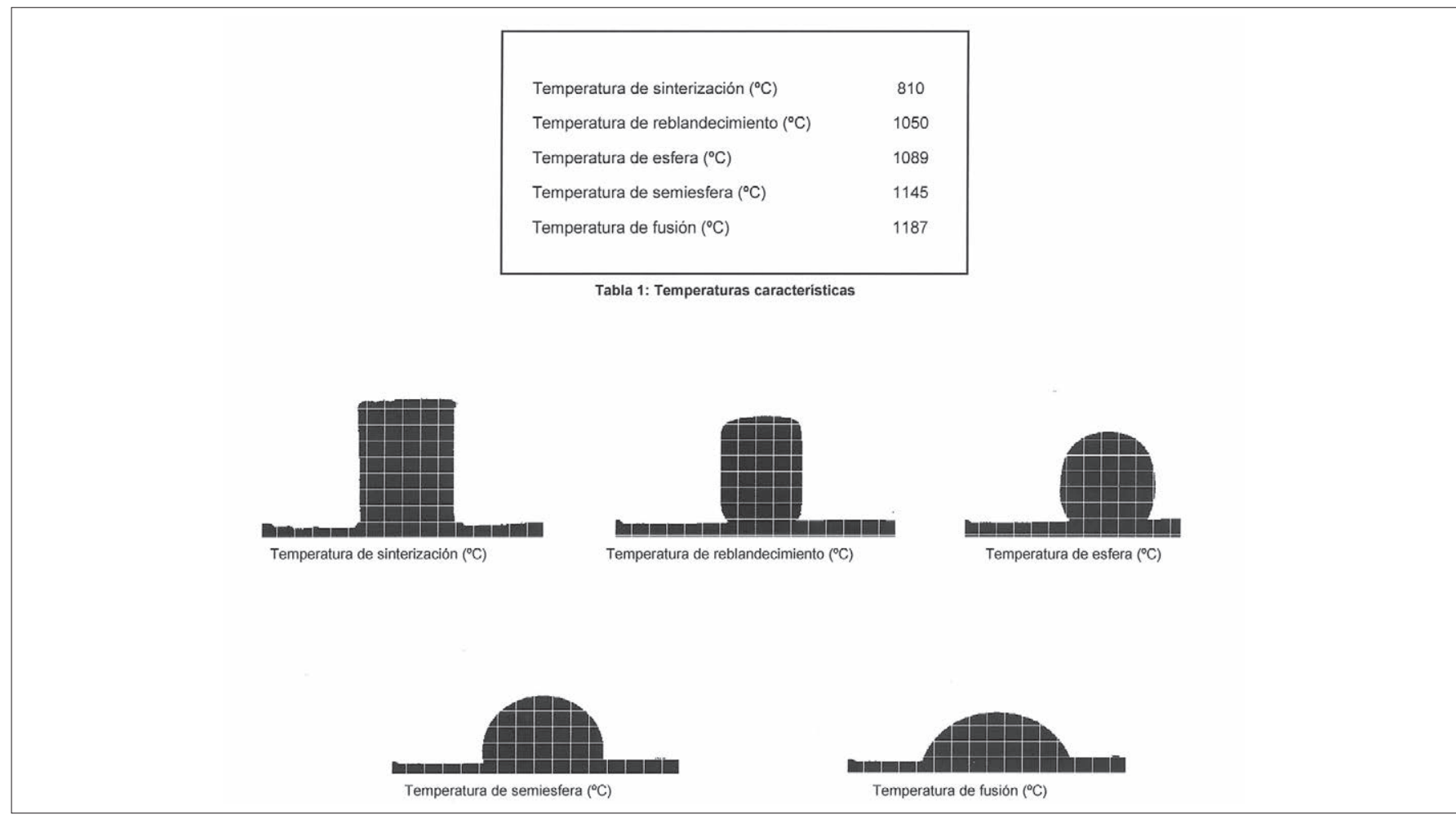

Figura 1. Etapas que caracterizan el comportamiento del material mediante microscopía de calefacción. 


\section{b. Efecto CLASSIC SILVER}

Para el desarrollo de este efecto metálico "noble", ha sido necesario regular la naturaleza fundente de la tinta desarrollada mediante la utilización de materiales cerámicos refractarios, posibilitando el desarrollo de efectos plateados a elevadas temperaturas.

Así pues, se ha favorecido y potenciado la generación de cristales orientados durante la etapa de tratamiento térmico para proporcionar, al incidir un haz de luz blanca, fenómenos de iriscéncia, como consecuencia de las interferéncias constructivas y destructivas producidas debido a los diferentes índices de refracción existentes entre los cristales y la matriz vítrea que los contiene.

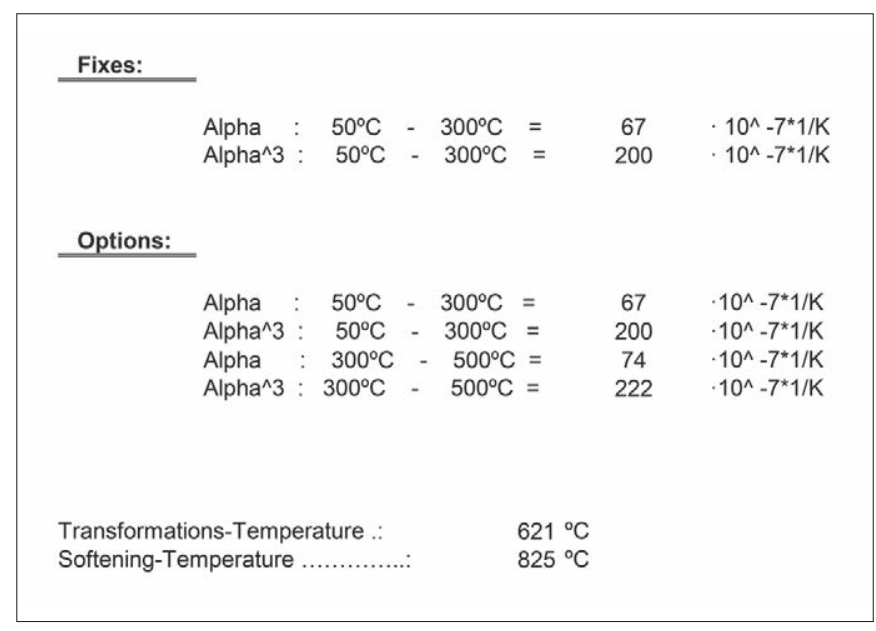

Figura 2. Valores obtenidos de la curva dilatométrica (expansión-contracción) característica el material fundente empleado en la tinta.

\section{c. Efecto CLASSIC OLDEN}

La obtención de este efecto óptico superficial ha sido posible gracias al empleo de materias primas capaces de cristalizar a elevadas temperaturas, proporcionando un efecto metálico envejecido. Para ello, ha sido necesario regular la reactividad de la tinta de modo que se obtuvieran composiciones estables a las temperaturas de trabajo.

Asimismo, y teniendo en consideración el pequeño tamaño que presentan las partículas que constituyen la tinta, también ha sido necesario potenciar el efecto por medio de la adición de precursores que faciliten la reacción del conjunto en los ciclos térmicos empleados.

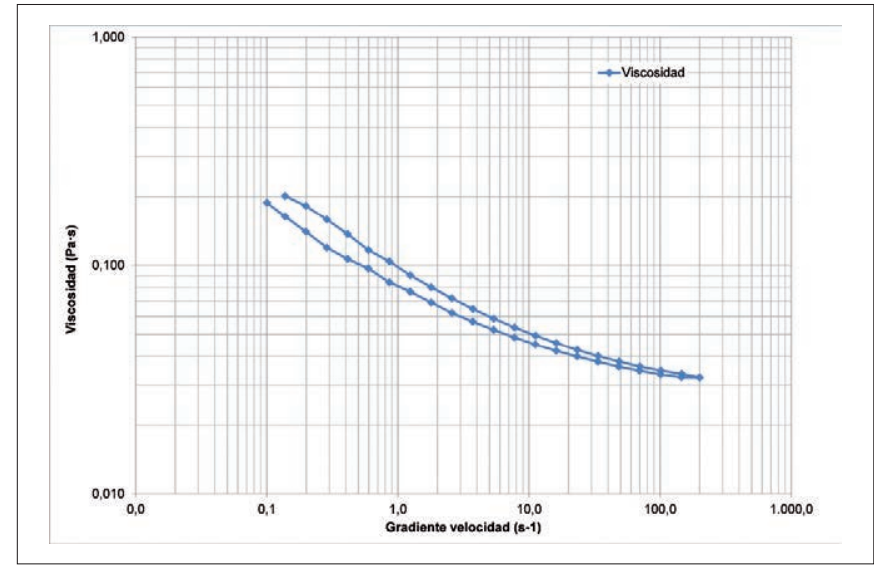

Figura 3. Comportamiento reológico (Curva de viscosidad) de la tinta Classic Olden a $30^{\circ} \mathrm{C}$.

\section{CONCLUSIONES}

Mediante el presente trabajo, se ha obtenido una nueva gama de efectos para la decoración de baldosas cerámicas fabricadas por monococción (revestimiento y pavimento), capaces de conferir, empleando la tecnología inkjet, propiedades tanto técnicas como estéticas diferenciadoras. Y es gracias a las infinitas posibilidades decorativas que ofrece esta tecnología y a los retos técnicos que es necesario vencer para la consecución de los objetivos propuestos, lo que confiere a la investigación llevada a cabo por Coloronda de un carácter innovador posibilitando el desarrollo de productos cerámicos de alto valor añadido.

La novedad de las posibilidades decorativas presentadas radica en el desarrollo de efectos ópticos de naturaleza metálica mediante el empleo de materias primas tradicionalmente utilizadas en el sector cerámico para la obtención, entre otros, de efectos "nobles" de naturaleza plateada y dorada, sin necesidad de emplear metales preciosos en la composición; hecho que comporta una reducción considerable de los costes de fabricación. Además, y de acuerdo con las ventajas técnicas y tecnológicas que supone la incorporación de la tecnología inkjet en el propio sistema productivo cerámico, hacen que la generación de tales efectos mediante el desarrollo de tintas acuosas sea posible, en toda o ciertas partes de las superficies cerámicas posibilitando la elaboración de infinidad de modelos diferentes.

Así pues, este trabajo sienta las bases de una nueva línea de investigación, en la que la generación de nuevos efectos ópticos superficiales de naturaleza metálica "noble" obtenida

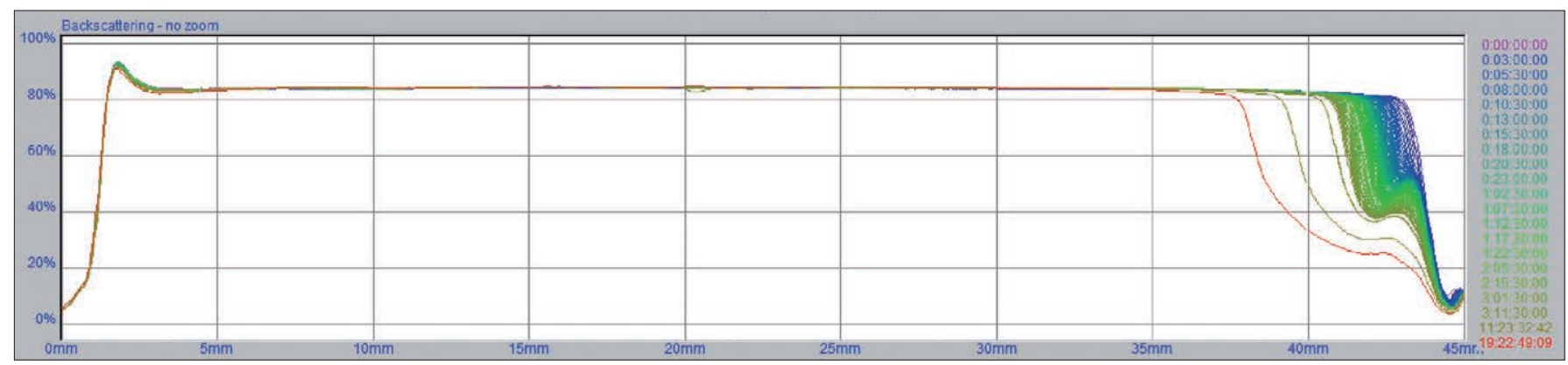

Figura 4. Perfil Backscattering de la tinta Classic Olden a $30{ }^{\circ} \mathrm{C}$ durante 19 días de envejecimiento. 


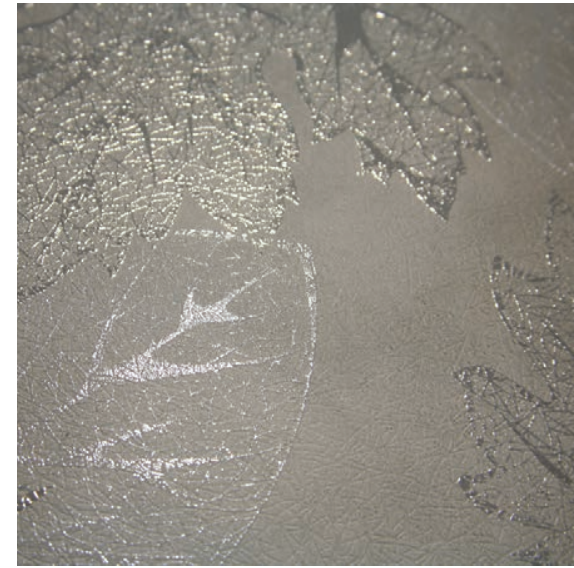

Efecto metálico "noble" plateado.

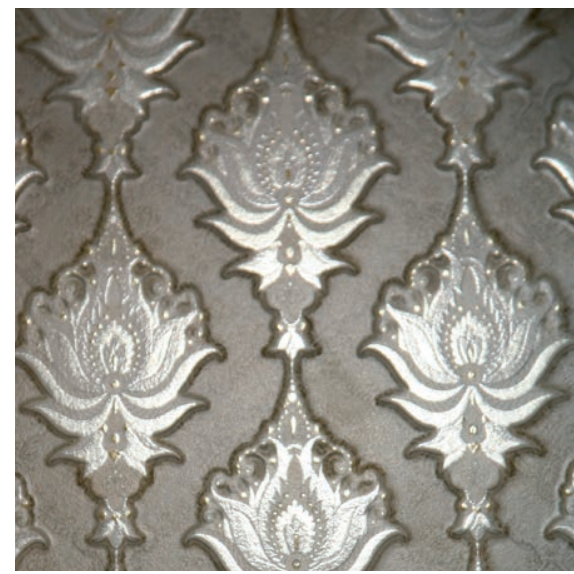

Efecto metálico "noble".

sobre sustratos cerámicos fabricados por monococción, cobra una gran importancia para la diversificación y diferenciación de productos dentro del sector cerámico.

Finalmente, ha sido demostrada la viabilidad técnica y reproducibilidad de los desarrollos obtenidos mediante la incorporación de las tintas formuladas en instalaciones actuales del sector.

A continuación se detallan algunas fotografías correspondientes a piezas cerámicas obtenidas mediante los efectos desarrollados.

\section{BIBLIOGRAFÍA}

1. Prado, M.; Fredericci, C.; Zanotto, E. Isothermal sintering with concurrent crystallization of polydispersed soda-lime-silica glass beads. Journal of NonCrystalline Solids 331 (2003) 145-156.

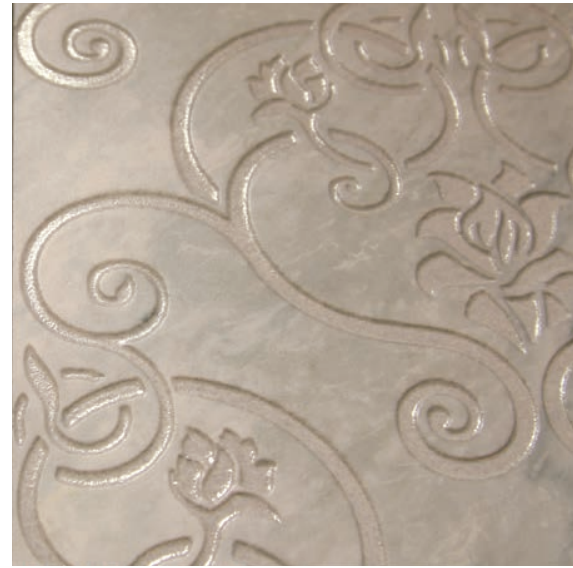

Efecto metálico "noble" plateado.

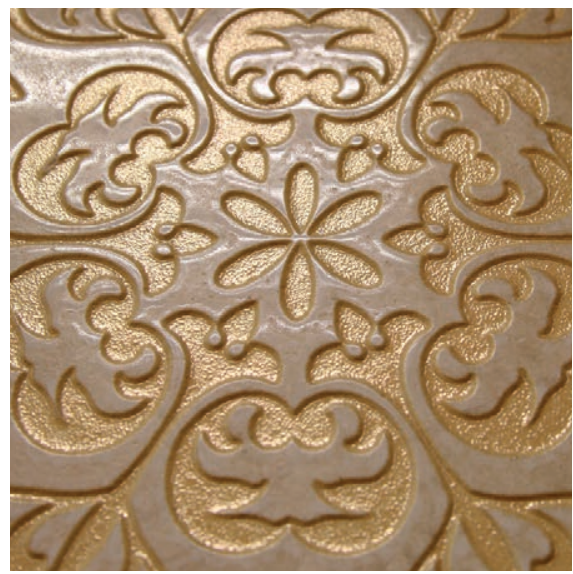

Efecto metálico "noble" dorado.

2. Prado, M.; Fredericci, C.; Zanotto, E. Non-isothermal sintering with concurrent crystallization of polydispersed soda-lime-silica glass beads. Journal of NonCrystalline Solids 331 (2003) 157-167.

3. Escribano, P.; et alt. Esmaltes y pigmentos cerámicos. Tomo 1. Faenza Editrice Iberica, S.L. Castellón (2001).

4. Siligardi, C.; et alt. Sintering behavior of Glass Ceramic Frits. The American Ceramic Society Bulletin (2000).

5. Randall, C.; Kim, N.; Kucera, J.; Cao, W.; Shrout, T. Intrinsic and Extrinsic Size Effects in Fine-Grained Morphotropic-Phase-Boundary Lead Zirconate Titanate Ceramics. J. Am. Ceram. Soc., 81 [3] (1998) 677-88.

6. Gribb, A.; Banfield, J. Particle size effects on transformation kinetics and phase stability in nanocrystalline TiO2. American Mineralogist, Volume 82, (1997) pages $717-728$

7. Cable, M. Classical glass technology. Materials Science and Technology, Vol 9. Cahn, R.W et alt. Weinheim-New York-Basel-Cambridge, VCH (1991).

8. Fernández Navarro, J.M. El vidrio. CSIF. Fundación Centro Nacional del Vidrio. Madrid (1991).

9. Rawson, H. Oxide glasses. Materials Science and Technology, Vol. 9. Cahn R.W. et alt. Weinheim-New York-Basel-Cambridge, VCH (1991). 